\title{
EMITTANCE GROWTH IN THE FEL RF-GUN*
}

\author{
M. Krassilnikov, S. Setzer ${ }^{\dagger}$, T. Weiland, Darmstadt University of Technology, Germany \\ A. Novokhatski, Stanford Linear Accelerator Center, California, USA
}

\section{Abstract}

A high brightness and low emittance is of crucial importance for the SASE-FEL at the TESLA Test Facility. Therefore a Photo-RF-Gun has been installed as particle source. Numerical simulations with codes like ASTRA [1] and MAFIA [2] show that the space charge dominated processes inside the RF-Gun contribute significantly to the emittance. In this paper we present the results of detailed studies with MAFIA TS2 which clarify the effects resulting in emittance growth for space charge dominated beams. It is shown that the resulting emittance can be minimized by changing the laser parameters like pulse length and spot size on the cathode. Additionally we present the concept of slice emittances which allows a more precise prediction of the real transverse emittance achievable with an emittance compensation scheme.

\section{SIMUATION PARAMETERS}

For the simulation of the beam dynamics inside the RFgun a two dimensional MAFIA TS2 [3] model of the complete structure including the external solenoids and a $25 \mathrm{~cm}$ drift tube has been developed. The electromagnetic field of the accelerating $\pi$-mode as well as the magnetic flux density of the solenoids were calculated using the eigenvalue and static solver modules of MAFIA. Table 1 gives an overview over the rf-gun parameters used for the simulations. Due to the sensitivity of the physical effects dominating the injection process to numercial errors all calculations were performed in double precision.

\begin{tabular}{|l|l|}
\hline Frequency & $1.3 \mathrm{GHz}$ \\
Acc. Gradient on Cathode & $50 \mathrm{MV} / \mathrm{m}$ \\
RF-Phase & $-32^{\circ}$ \\
Laser Pulse Length & $10.3 \mathrm{ps}$ (flat-top) \\
Laser Rise/Fall Time & $2 \mathrm{ps}$ \\
Laser Radius & $1.5 \mathrm{~mm}$ \\
Max. Magnetic Flux Density & $0.2 \mathrm{~T}$ \\
Long. Solenoid Position & $12.5 \mathrm{~cm}$ \\
Total Bunch Charge & $1 \mathrm{nC}$ \\
\hline
\end{tabular}

Table 1: RF-Gun Simulation Parameter

\section{SLICE EMITTANCE}

The transverse emittance of a bunch is usually calculated by projecting the transverse phase space of all particles on a single plane. The projected emittance is then calculated

\footnotetext{
* Work supported in part by DESY and DFG (FOR 272/2-1)

$\dagger$ setzer@temf.de
}

with the help of equation 1.

$$
\varepsilon_{p}=\sqrt{\overline{x^{2} x^{\prime 2}}-{\overline{x x^{\prime}}}^{2}}
$$

A more precise description of the transverse phase space volume can be given by subdividing the bunch of the total length $\sigma$ longitudinally into several slices. In this case the transverse slice emittance is given by

$$
\varepsilon_{s}=\frac{1}{\sigma} \int_{-\sigma / 2}^{\sigma / 2} \varepsilon_{p}(z) d z
$$

where $\varepsilon_{p}(z)$ is the projected emittance of the particles associated with the beam slice at position $z$. Compared to the projected emittance all longitudinal correlations between the individual slices are excluded due to the modified calculation methode. The idea of the emittance compensation scheme as proposed in [4] is to eliminate these longitudinal correlations. Therefore the slice emittance represents the lower limit for the achievable transverse beam emittance.

\section{SIMULATION RESULTS}

\subsection{Emittance development}

The development of the longitundinal and transverse emittances during the passage through the RF-Gun with additinal drift tube is shown in fig. 1. At injection the bunch has a transverse emittance equal to zero. The strong

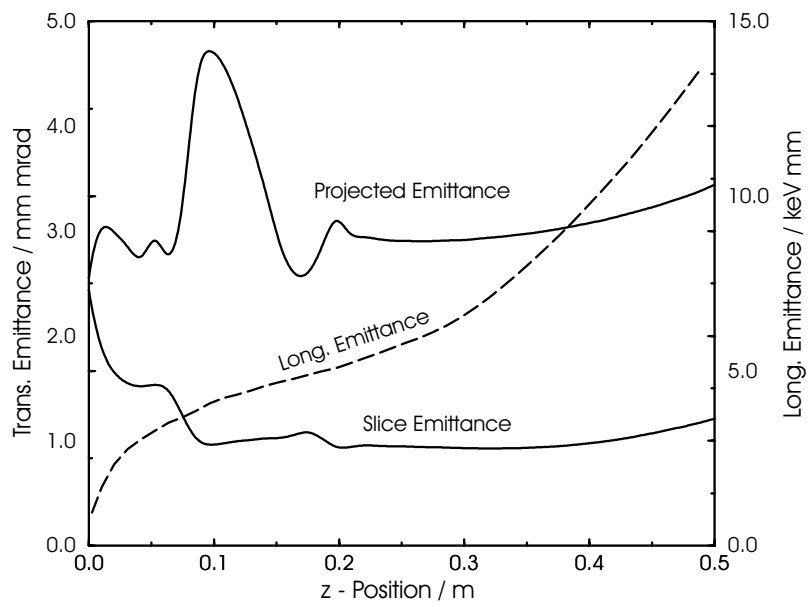

Figure 1: Emittance development inside the RF-Gun with additional drift tube

variation of the transverse projected emittance is caused by a permanently changing longitudinal correlation between individual beam slices due to space charge and external 
fields. The slice emittance is not affected by those correlations therefore it remains nearly constant. The phase ellipses for head, middle and tail slices at three different positions inside the RF-Gun are shown in Fig. 2 illustrating that the change of the projected emittance is only caused by different orientations of the individual phase ellipses. This rotation of the ellipses is due to the external solenoidal magnetic field. After passage through this magnetic field the phase space ellipses are arranged as required for the emittance compensation methode. It can also be observed
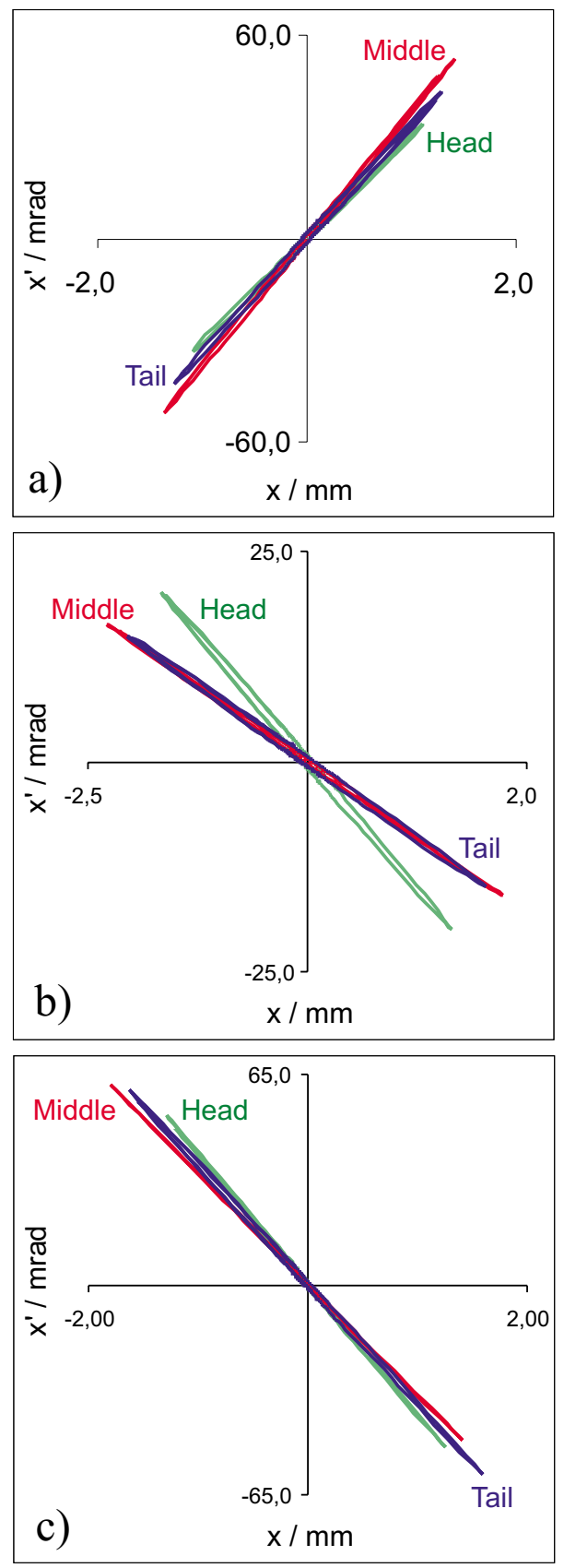

Figure 2: Phase space ellipses (head, middle and tail) at three different longitudinal positions. a) $z=4.2 \mathrm{~cm}$, b) $z=10.7 \mathrm{~cm}, \mathrm{c}) z=16.4 \mathrm{~cm}$

that the main contribution to the transverse emittance arises during the injection process in the area close to the cathode. To get a better insight into the physical background of the emittance formation the dynamics inside an individual transverse layer in the vicinity of the cathode was studied. The result for the transverse phase space at three different times is shown in Fig. 3. The change in the transverse

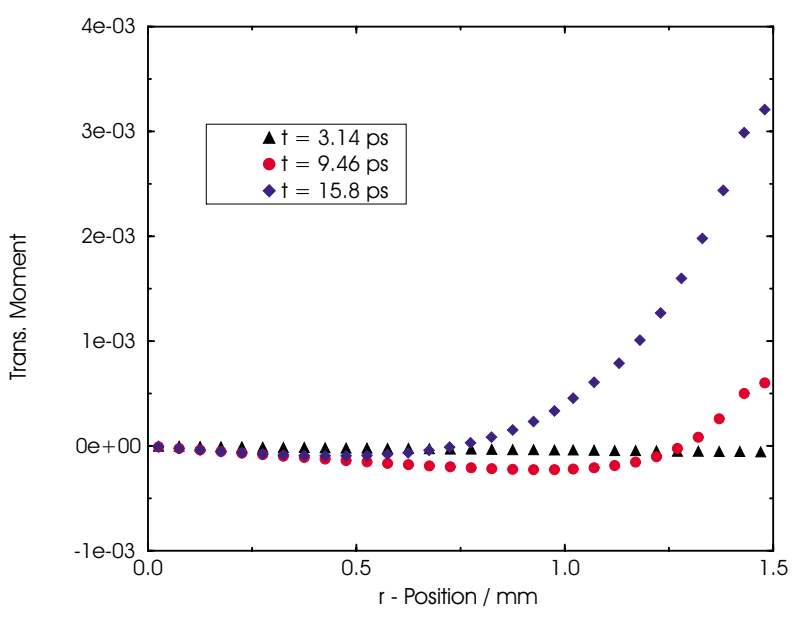

Figure 3: Emittance formation inside a single layer

phase space is the result of two opposing forces acting on the beam particles namely the focusing force of the accelerating $\mathrm{rf}$ field and the defocusing force of the space charge fields. The resulting radial component of the Lorentz force acing on the particles inside the layer is shown in Fig. 4 a-c. Right after injection (Fig. 4 a) mainly the focusing effect of the azimuthal magnetic field of the rf-mode contribute to the resulting Lorentz force. This force is linear in radial direction inside the beam, thus the transverse phase space of the particles is only rotated but not distorted. At this moment the space charge fields just start to formate and can not severly impact the transverse emittance by increasing the phase space volume. With more time elapsed after injection the defocusing space charge force becomes the dominating part of the resulting Lorentz force as depicted in fig. $4 \mathrm{~b}$ and c. This force is strongly non-linear in radial direction thus leading to transverse phase space distortion resulting in emittance growth. The main reason for the non-linear behaviour of the space charge force is the small bunch length, the bunch has comparable longitudinal and transverse dimensions of about two milimeter rms.

This emittance growth takes place inside each individual beam slice and is mainly independent of the longitudinal position inside the bunch, therefore the resulting emittance can not be reduced by eliminating longitudinal correlations with an emittance compensation scheme.

\subsection{Parameter optimization}

In order to reach a higher beam quality the influence of the laser parameters such as pulse length and laser spot size on the cathode has been investigated. The results for the for the transverse projected as well as slice emittance are shwon in Fig. 5. It is possible to decrease the final 

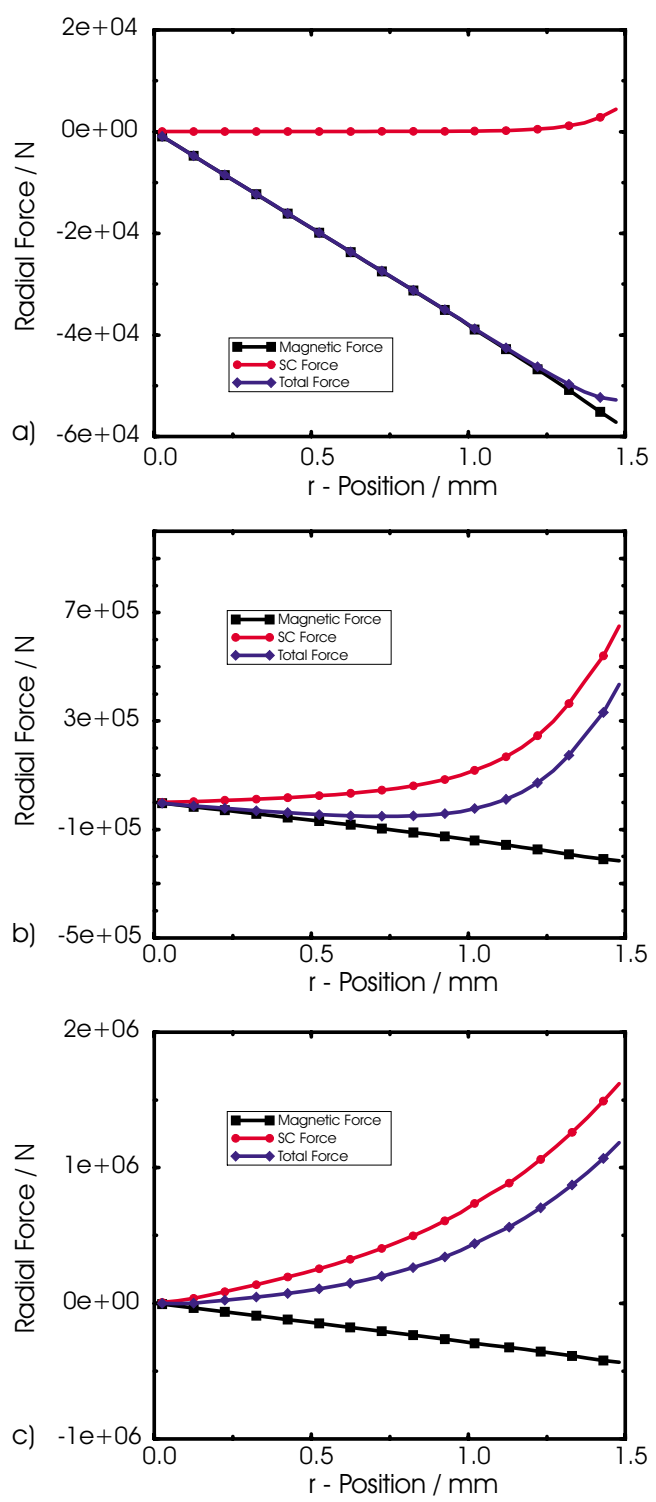

Figure 4: Lorentz forces acting on the particles inside one layer. a) $t=3.15 \mathrm{ps}$, b) $t=9.46 \mathrm{ps}$, c) $t=15.8 \mathrm{ps}$

transverse emittance behind the rf-gun by increasing the laser pulse duration while keeping the total bunch charge constant. This leads to a much longer bunch reducing the non-linearity as well as the strength of the space charge effects. On the other hand the increased laser pulse duration results in additional longitudnal emittance growth as depicted in Fig. 6 which may lead to unwanted additional energy spread during the subsequent acceleration.

\section{CONCLUSION}

It was shown that the major portion of the emittance growth in the FEL RF-Gun arises in the vicinity of the cathode. This is caused by the superposition of the nonlinear space charge forces and the linear focusing forces of the rf-field inside the bunch. The behaviour of the projected emittance is a result of changing longitudinal corre-
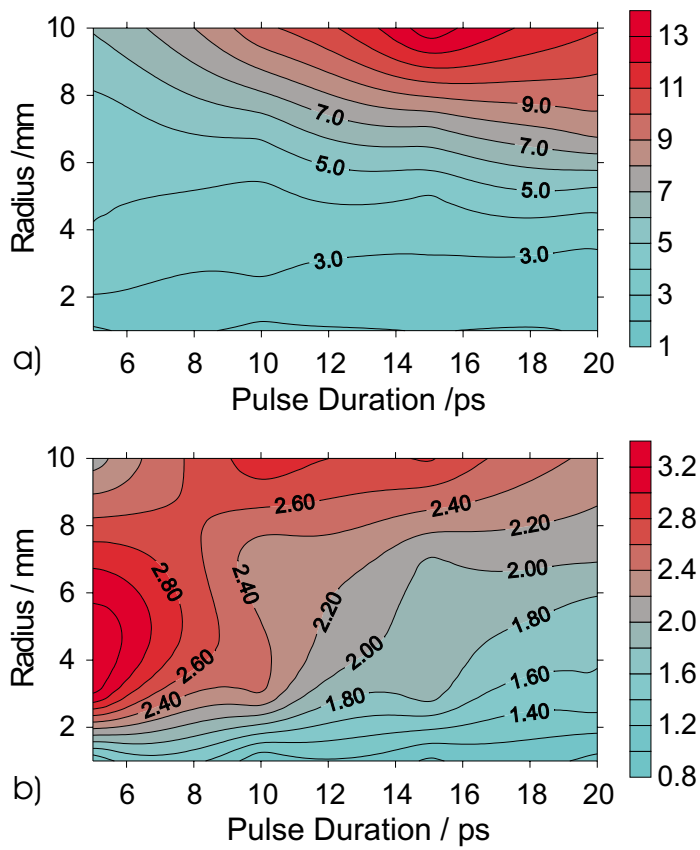

Figure 5: Transverse emittances (mm-mrad) for different laser parameters. a) Projected Emittance, b) Slice Emittance.

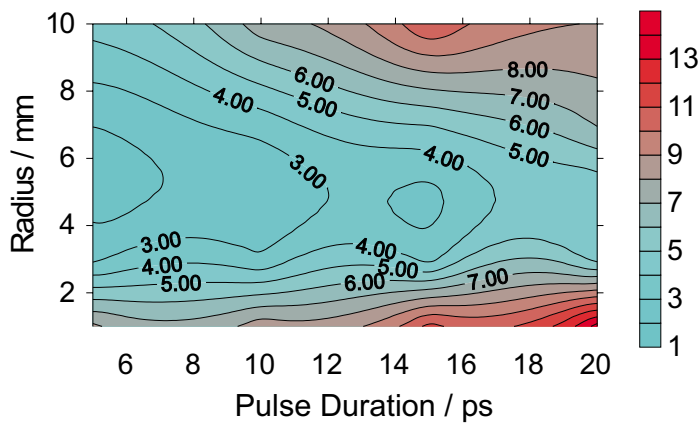

Figure 6: Longitudinal emittance (keV-mm) for different laser parameters.

lations inside the bunch. Nevertheless the slice emittance remains constant representing the lower limit for the achivable transverse beam emittance. Further improvement of the transverse beam quality is possible by optimizing the laser parameters but is limited by the longitudinal phase space properties.

\section{REFERENCES}

[1] M. Ferarrio et al., "Conceptual Design of the TESLA XFEL Photoinjector", DESY TESLA-FEL 01-03, 2001

[2] M. Zhang, P. Schütt, "TESLA FEL Gun Simulations with PARMELA and MAFIA", Proc. of the CAP, 1996, pp. 21-26.

[3] CST GmbH, Büdinger Strasse 2a, D-64289 Darmstadt

[4] B. E. Carlsten, "New Photoelectric Injector Design for the Los Alamos National Laboratory XUV FEL Accelerator", Nucl. Instrum. Methods A 285, p313, 1989 